\title{
Comprendre les antécédents de la satisfaction des clients envers l'achat en ligne : une étude empirique de la théorie de l'autodétermination
}

\author{
Urvashi Tandon $^{a}$, Ravi Kiran ${ }^{b}$
}

RÉSUMÉ. Cet article vise à comprendre les indices de la satisfaction des clients en ce qui concerne l'achat en ligne en Inde en utilisant la théorie de l'autodétermination (TDA). Cette recherche valide le plaisir perçu, l'influence sociale, les interactions sur les réseaux sociaux, la logistique inverse et le paiement à la livraison (pay on delivery ou POD) en tant que nouveaux indices de la satisfaction des clients pour l'achat en ligne. Les données ont été recueillies au moyen d'un questionnaire autoadministré et structuré ciblant les acheteurs en ligne dans les États de l'Inde du Nord. Un échantillon de 424 acheteurs en ligne a été validé à l'aide de la modélisation d'équations structurelles (SEM). Les conclusions de l'article révèlent que l'influence sociale, la logistique inverse et le paiement à la livraison (POD) ont eu un impact positif et significatif sur la satisfaction des clients. Le plaisir perçu apparait comme étant le meilleur indice de l'achat en ligne. Cependant, les interactions sur les réseaux sociaux apparaissent comme un indice négligeable de la satisfaction des clients. Cette recherche constitue l'un des efforts initiaux qui valident empiriquement le POD, les interactions sur les réseaux sociaux, l'influence sociale, la logistique inverse et le plaisir perçu dans le contexte de l'achat en ligne en utilisant la TDA. Grâce à cette étude, les cybercommerçants qui se préparent à étendre leurs activités en Inde obtiennent des informations importantes sur les moteurs de l'achat en ligne menant à la satisfaction des clients. En retour, cela leur permettra de développer des stratégies de marketing et de les mettre en place pour cibler l'énorme marché inexploité.

ABSTRACT. This paper aims to help understand the predictors of customer satisfaction with respect to online shopping in India, by using the Self-Determination Theory (SDT). This research validates perceived enjoyment, social influence, social media interactions, reverse logistics and pay on delivery (POD) mode of payment as new predictors of customer satisfaction in online shopping. Data was collected through a selfadministered and structured questionnaire targeting online shoppers in North India states. A sample of 424 online shoppers was considered validated in this research using Structural Equation Modelling (SEM). The findings of the paper revealed that social influence, reverse logistics and POD mode of payment had a significant positive impact on customer satisfaction. Perceived enjoyment emerged as the strongest predictor of online shopping. However, social media interactions emerged as an insignificant predictor of customer satisfaction. This research is one of initial endeavour in online shopping that empirically validated POD, social media interactions, social influence, reverse logistics, and perceived enjoyment by using the SDT. Through this study, online retailers preparing to expand their operations in India have important insights regarding the drivers of online shopping leading to customer satisfaction. This in turn will help in developing marketing strategies and their implementation to target the buge untapped market.

\section{Introduction}

L'achat en ligne a pris de l'ampleur au cours des dernières années en raison de facteurs comme la percée de l'Internet, la commodité de l'accès en tout temps, l'accès rapide aux renseignements et l'avis en ligne reliés au produit, mais surtout en raison du mode de paiement nommé paiement à la livraison (pay on delivery ou POD) en Inde. On peut en témoigner grâce au paiement de 2,8 milliards de roupies indiennes (INR)

\footnotetext{
a Professeure agrégée en marketing, Université Chitkara, Penjab, Inde

b Professeure titulaire en administration des affaires, Université Thapar, Patiala, Inde
} 
par POD en Inde (Deloitte et Assocham India, 2014). La percée d'Internet en Inde a atteint environ 665 millions de personnes en 2019 et augmentera à 829 millions en 2021. Or, de ce nombre, seulement 20 millions sont des acheteurs en ligne actifs qui effectuent des achats mensuels (Vijay, 2020). L'industrie du commerce électronique indien dépassera les États-Unis d'ici 2034 avec 64 milliards de dollars américains en 2020 et 200 milliards de dollars américains en 2026 (IBEF, 2018). Les commerçants en ligne font face au défi logistique de livrer des produits dans de vastes zones géographiques de l'Inde, qui regroupent 6000 petites villes et 600000 villages (Nielsen, 2017).

Ces chiffres révèlent clairement que, même si la percée de l'Internet est en forte augmentation, cela n'a pas produit une augmentation du cybercommerce (Sushma, 2017) et qu'il y a encore beaucoup d'occasions pour les cybercommerçants de cibler les secteurs qui seront éventuellement en ligne. Un cadre global qui intègre les meilleures pratiques de gouvernance, tout en insistant sur les besoins uniques de cette vaste base de consommateurs autonomes doit être élaboré. Les principaux défis auxquels les commerçants en ligne font face pour percer dans le marché indien comprennent l'aspect exotique de l'utilisation de systèmes numériques, des infrastructures irrégulières et la grande variété de langues et de dialectes régionaux (Vijay, 2020). Les principales raisons de la performance chancelante sont les frais de renvoi à l'origine en Inde (return to origin ou RTO), en particulier pour les commandes avec paiement à la livraison (POD) ou avec un envoi contre remboursement (cash on delivery ou COD). Ainsi, c'est une exigence essentielle que d'étudier les variables qui peuvent faciliter l'acquisition de consommateurs pour les commerçants en ligne.

Un client vigilant évalue la rétroaction sur les produits à partir de sources telles que ses amis et les pairs, et tente d'évaluer l'authenticité des promesses faites par un cybercommerçant avant de faire un achat en ligne. Dans ce contexte, en savoir plus sur les variables qui conduisent à un achat et à un rachat devient impératif. Les commentaires et les évaluations de produits en ligne, les informations et la persuasion s'avèrent avoir un lien significatif et positif sur les intentions d'achat des consommateurs et sur leur satisfaction (Ashman et al., 2015; Zhang et al., 2014).

De manière similaire, la logistique inverse (qui regroupe toutes les procédures liées au retour d'un produit, aux réparations et à l'entretien) a transformé l'appréhension concernant le fait de recevoir des produits défectueux lors d'achats en ligne. Les politiques de retour justes et modérées ont un impact positif sur les comportements d'achat en ligne (Oghazi et al., 2018). De même, les influences sociales qui précisent les relations dans un groupe de membres ont été validées en tant que facteur déterminant conduisant à un achat en ligne (Tandon et al., 2017).

Par conséquent, la recherche actuelle couvre divers facteurs autres que le plaisir perçu, qui a été validé par des modèles antérieurs. La plupart de ces concepts ont été étudiés en tant que concepts indépendants, mais leur incidence sur la satisfaction des clients en comparaison avec d'autres n'a pas réellement été validée pour l'achat en ligne. Afin de combler cette lacune, la théorie de l'autodétermination (TDA) (Deci et Ryan, 1985) est employée dans cette recherche pour soutenir l'impact de la logistique inverse, des réseaux sociaux, de l'influence sociale, du POD et du plaisir perçu sur la satisfaction des clients.

Cette étude comprend de nombreuses contributions théoriques et pratiques. En plus d'augmenter la connaissance sur la satisfaction des clients par la validation de la TDA, elle contribue également à mettre en place des pratiques durables pour les commerçants en ligne et pour les consommateurs. Se concentrer sur des concepts comme le POD, les interactions sur les réseaux sociaux, l'influence sociale, le plaisir perçu et la logistique inverse ne soutient pas seulement les commerçants en ligne pour répondre aux besoins des clients, mais également pour répondre aux demandes et besoins changeants des clients, ce qui augmente ainsi le niveau de satisfaction de ces derniers. Le commerce électronique soutient la culture numérique, l'inclusion financière et la facilité à faire des affaires, ce qui entraîne de la création d'emplois et une croissance économique intelligente, qui, en retour, mène au développement du territoire.

\section{Contexte théorique et hypothèses de développement}

\subsection{Théorie de l'autodétermination}

La théorie de l'autodétermination (TDA) proposée par Deci et Ryan (1985) met en évidence les problèmes liés aux motivations intrinsèques et extrinsèques. La TDA est axée sur la façon dont les humains intègrent la conscience globale du soi en 
régulant leurs besoins psychologiques pour leur bienêtre et leur satisfaction (Hwang, 2010; Gagné et Deci, 2005). Les chercheurs ont confirmé que la théorie de l'autodétermination met l'accent sur les motivations intrinsèques (plaisir perçu) et sur les motivations extrinsèques (normes sociales) comme instincts comportementaux dans le comportement général (Hwang, 2010).

La TDA a été abondamment validée par les chercheurs en psychologie sociale, mais ce modèle n'a pas été étudié en profondeur dans le domaine du commerce en ligne (Hwang, 2010; White, 2015; Gao et al., 2018). En raison des besoins croissants pour les achats en ligne, les sites web des cybercommerçants présentent un certain nombre de signaux et de motivateurs afin de rendre leur expérience d'achat agréable et afin de répondre à leurs besoins. Quelques études précédentes ont évalué certains besoins psychologiques (Kim et Drumwright, 2016; Martin et Hill 2012). Cette recherche vise à déterminer quels besoins motivationnels en particulier sont actifs lors de l'achat en ligne et lesquels conduisent à la satisfaction du client lorsqu'il fait de tels types d'achats.

Le plaisir perçu a été classé en tant que motivation intrinsèque par Venkatesh et Speier (2000), tandis que l'influence sociale a été classée en tant qu'effet positif significatif sur les intentions d'achat (Venkatesh et al., 2003; Hwang, 2010). Slyke et al. (2002) ont confirmé que l'achat en ligne a été considéré comme étant une activité sociale pratiquée par les femmes, plutôt que comme l'adoption de la technologie. La théorie unifiée de l'acceptation et de l'utilisation de la technologie 2 (UTAUT2) (Venkatesh et al., 2012) conclut également que l'influence sociale et les motivations hédonistes (plaisir perçu) prédisent de manière importante les intentions d'acheter en ligne. Recevoir de la rétroaction des pairs, lire des avis sur les produits et discuter dans des communautés grâce à des interactions sur les réseaux sociaux répondent aux besoins psychologiques de base, ce qui conduit à l'adoption d'une technologie et à la satisfaction envers cette dernière (Gao et al., 2018). De même, le mode de paiement par POD (Tandon et al., 2017) et les politiques de retour (Oghazi et al., 2018) peuvent être considérés comme des facteurs de motivation importants pour l'achat en ligne. En effet, le POD, les politiques de retour et les interactions sur les réseaux sociaux peuvent répondre aux besoins psychologiques.

Revue Organisations \& Territoires $•$ Volume $29 \cdot \mathrm{N}^{\circ} 3 \cdot 2020$
Les besoins psychologiques sont «ces besoins qui, lorsque satisfaits (ou entravés), sont normalement bénéfiques (ou défavorables) à la croissance psychologique continue, au développement et au bien-être d'une personne» (Deci et Ryan 2000, p. 229). Ainsi, les individus pratiquent les activités qui conduisent à l'estime et à l'amélioration de soi.

De leur côté, les commerçants en ligne intègrent certaines fonctionnalités sur leur site web telles que des politiques de retour, des forums de discussion, le POD, des commentaires et évaluations sur les produits ainsi qu'une navigation exempte de produits, qui nourrissent les besoins psychologiques de base. Ces fonctionnalités d'achat en ligne motivent les consommateurs à effectuer un achat et apportent des résultats positifs conduisant à la satisfaction.

La TDA a également été étudiée précédemment dans le cadre d'achat en ligne par d'autres chercheurs (Hew et Kadir, 2017; Gao et al., 2018; Hwang, 2010; White, 2015). Ces recherches ont prouvé le rôle critique de la satisfaction des besoins pour générer des résultats positifs. D'abord, Hew et Kadir (2017) ont étudié des environnements d'apprentissage virtuel infonuagiques et ont constaté l'impact significatif des besoins psychologiques sur le comportement face à l'utilisation. Hwang (2010) a utilisé la TDA et a constaté un impact positif important des normes sociales et du plaisir perçu sur le comportement. Gao et ses collaborateurs (2018) ont présenté une nouvelle perspective de la TDA en validant le rôle de la téléprésence et de la présence sociale, qui influencent les intentions d'achat. Enfin, White (2015) a employé la TDA et a corroboré l'impact des motivations intrinsèques et extrinsèques sur la satisfaction des clients.

Dans l'ensemble, ces études sont axées sur la satisfaction des besoins, qui est indispensable pour stimuler l'achat en ligne. Ainsi, en se basant sur la littérature, cette étude présente la TDA pour expliquer l'impact du plaisir perçu, de la logistique inverse, du POD, de l'influence sociale et des interactions sur les réseaux sociaux pour le marché de l'achat en ligne.

\subsection{Satisfaction des clients}

La satisfaction des clients est une variable dépendante largement étudiée dans le contexte de l'achat en ligne. Elle est définie comme étant la réponse de satisfaction du consommateur: "Il s'agit d'un jugement sur un produit ou un élément du service, ou le produit du service lui-même, qui a offert (ou qui offre) un niveau 
agréable de satisfaction liée à la consommation, incluant les niveaux de sous-satisfaction ou sursatisfaction» (Oliver, 1993, p. 420). La satisfaction des clients joue un rôle pertinent dans l'achat en ligne, car elle influence la décision des consommateurs à continuer de faire des achats en ligne ou non. Elle a été étudiée de manière approfondie en tant que variable dépendante de l'achat en ligne (Crisafulli et Singh, 2017). Toutefois, la plupart des conclusions des recherches préconisent l'impact des influences individuelles et psychologiques menant à des répercussions importantes sur la théorie de la satisfaction des clients et sa pratique (Crisafulli et Singh, 2017; Tandon et al., 2017).

Ainsi, il est indispensable de comprendre les variables qui influencent la satisfaction des clients. Du point de vue de la TDA, la motivation est un continuum et elle varie selon les comportements contrôlés par les exigences externes et par les tâches accomplies pour obtenir satisfaction (Deci et Ryan, 2000; White, 2015; Kumar et Kashyap, 2018). White (2015) s'est servi de la TDA et a confirmé que la motivation est un facteur déterminant de la satisfaction.

Pour approfondir la compréhension de la satisfaction des clients en utilisant les concepts de la TDA, cette étude étend le cadre théorique de Hwang (2010), où le plaisir perçu et les normes sociales ont été validés en tant que principaux facteurs de motivation. Cette étude étend le modèle aux interactions sur les réseaux sociaux, au POD et à la logistique inverse comme principaux facteurs de motivation extrinsèque conduisant à la satisfaction des clients.

\subsection{Plaisir perçu}

Le plaisir perçu se définit comme étant « l'amusement ou le plaisir découlant de l'utilisation d'une technologie, et il a été démontré qu'il joue un rôle dominant dans la détermination de l'acceptation de la technologie et de son utilisation » (Brown et Venkatesh, 2005, p. 400). Les études précédentes (Venkatesh et al., 2012; Thong et al., 2006; Sakshi et al., 2020) ont conceptualisé le plaisir perçu comme un facteur essentiel motivant les consommateurs à faire des achats en ligne. Le plaisir perçu est une motivation intrinsèque qui représente la mesure dans laquelle le plaisir est le résultat de l'utilisation de l'informatique (Chang et Chen, 2015). L'étude de Hwang (2010), tout en comparant l'attitude des hommes et des femmes qui font des achats en ligne, a confirmé que le plaisir perçu obtient plus d'impact sur les acheteurs en ligne masculins. Selon la théorie de l'autodétermination (TDA) et la théorie de l'action raisonnée (TRA), les motivations intrinsèques telles que le plaisir perçu sont des facteurs déterminants importants de l'achat en ligne. Par conséquent, l'hypothèse suivante est proposée :

\section{H1 : Il existe une association positive entre le plaisir perçu et la satisfaction des clients.}

\section{$1.4 \quad$ Mode de paiement par POD (paiement à la livraison)}

En Inde, les clients doivent fournir les détails de la carte de crédit/débit pour commander en ligne. Les clients indiens qui se méfient des paiements en ligne avec une carte de crédit/débit auraient un comportement contrôlé envers l'achat en ligne. L'utilisation limitée des cartes de crédit/débit a convaincu les cybercommerçants d'offrir un substitut sous la forme d'un paiement non électronique, c'est-à-dire le mode de paiement envoi contre remboursement (ECR), qui a instauré la confiance chez les clients indiens et qui a réduit, dans une certaine mesure, l'appréhension envers les produits défectueux puisque les clients ne paient qu'après avoir reçu le produit.

En novembre 2016, la démonétisation faite par le gouvernement en place a déplacé toute l'attention sur la numérisation. Par la suite, les commerçants en ligne ont également adapté l'ECR en offrant des paiements par carte de débit, mais après que le produit ait été livré (POD). L'économie indienne est fondée sur la monnaie, et de tels changements dans les modèles et évaluations culturels nécessitent du temps et des ressources. Le POD a convaincu les commerçants en ligne grâce à ses livraisons en temps voulu, ce qui offre ainsi un service amélioré qui augmente la satisfaction des clients. En outre, quelques études indiennes comme celle de Tandon et ses collaborateurs (2017) ont recommandé l'utilisation du mode de paiement ECR pour l'achat en ligne, mais le concept n'est pas bien établi dans la littérature. L'étude de Chiejina et Olamide (2014) sur les clients nigériens souligne que le POD est un vecteur de confiance important entre les clients et les commerçants en ligne. Bien que suggéré et considéré dans la plupart des études, le POD n'a pas été abondamment validé de manière empirique en tant que concept. Pour combler cette lacune, il a été 
inclus dans la présente étude. Comprenant l'importance de ce mode de paiement et avec le soutien de la littérature, l'hypothèse suivante est proposée :

\section{H2: Il existe une association positive entre le mode de paiement par POD et la satisfaction des clients.}

\subsection{Interactions sur les réseaux sociaux}

Les réseaux sociaux sont des services en ligne, aussi connus sous le nom de "sites de réseautage social», qui font référence à des réseaux de relations et d'interaction entre différents usagers (groupes ou individus) (Kempe et al., 2003; Sheikh et al., 2019). Les interactions sur les réseaux sociaux influencent également le comportement du consommateur, de l'acquisition d'information sur le produit jusqu'au comportement après l'achat du produit (Duffett, 2015). Un message positif diffusé grâce à des interactions sur les réseaux sociaux augmente les ventes du produit. Ainsi, les interactions sur les réseaux sociaux ont été validées dans cette étude comme étant un facteur de motivation.

Hajli (2014) souligne l'incidence positive importante des interactions sur les réseaux sociaux sur les intentions d'achat des clients. Muda et al. (2016) ont évalué les comportements d'achat en ligne de la génération $\mathrm{Y}$ en Malaisie et ont constaté que ces achats étaient effectués en majorité chez des cybercommerçants qui fonctionnent par l'entremise de Facebook et d'Instagram. Les clients utilisent les interactions sur les réseaux sociaux comme une plateforme pour exprimer leurs convictions, mais surtout pour accéder à de l'information sur les produits (Duffett, 2015; Ioanăs et Stoica, 2014). De plus, les évaluations sur les produits en ligne émises par différentes couches d'utilisateurs et évoluant grâce aux interactions sur les réseaux sociaux ont eu les répercussions les plus importantes sur la commercialisation. L'incapacité d'un commerçant en ligne à résoudre le problème a un impact négatif sur la satisfaction des clients, ce qui conduit ainsi à une diminution des intentions de rachat et du bouche-àoreille favorable (Crisafulli et Singh, 2017). Sur la base de ce qui précède, l'hypothèse suivante est formulée :

\section{H3 : Il existe une association positive entre les interactions sur les réseaux sociaux et la satisfaction des clients.}

\subsection{Influence sociale}

L’influence sociale a été définie comme étant «la mesure dans laquelle les clients perçoivent que les personnes importantes (p. ex., famille et amis) croient qu'ils devraient se servir d'une technologie en particulier» (Venkatesh et al., 2012, p. 159). Des études précédentes (Tandon et Kiran, 2019; Hwang, 2010) ont confirmé une association positive importante de l'influence sociale sur le comportement d'achat en ligne. Au contraire, l'étude de Dharmawirya et Smith (2012) a confirmé l'impact négligeable de l'influence sociale sur les consommateurs indonésiens. Ainsi, il peut être démontré que les conclusions ne sont pas cohérentes. En conséquence, l'hypothèse suivante est proposée :

\section{H4 : Il existe une association positive entre l'influence sociale et la satisfaction des clients.}

\subsection{Logistique inverse}

En matière de satisfaction des clients, un moyen rapide et performant permettant aux clients de retourner les produits défectueux est presque aussi important que le moyen de livraison. Harris et Martin (2014) ont conclu que la logistique inverse a inspiré la confiance chez les clients qui achètent en ligne. Cette étude met aussi l'accent sur l'utilisation efficace de systèmes tierce partie. Pour obtenir l'avantage concurrentiel et pour rester compétitifs sur les marchés, la plupart des commerçants en ligne offrent une politique de retour permissive ( $\mathrm{Li}$ et al., 2013; Altug et Aydinliyim, 2016). Les procédures de retour aident également à réduire l'appréhension envers les produits défectueux. Une politique de retour permissive adoptée par les commerçants en ligne est un signe de la qualité du service et entraîne ainsi une augmentation des ventes (Tavana et al., 2019; Pei et al., 2014). Oghazi et al. (2018) ont confirmé les répercussions positives importantes d'une politique de retour indulgente sur les intentions d'achat. Pei et al. (2014) soulignent que ce type de politique renforce la confiance envers l'entreprise. Par conséquent et sur la base de ce qui précède, l'hypothèse proposée est la suivante :

\section{H5 : Il existe une association positive entre la logistique inverse et la satisfaction des clients.}

Sur la base de la littérature, dans la présente étude, le plaisir perçu, les interactions sur les réseaux sociaux, la logistique inverse et le POD sont des variables indépendantes conceptualisées, et elles ont un impact positif sur la satisfaction des clients. 
À partir de ce qui a été discuté précédemment, un modèle théorique est proposé. La figure 1 résume le

modèle conceptuel de l'étude.

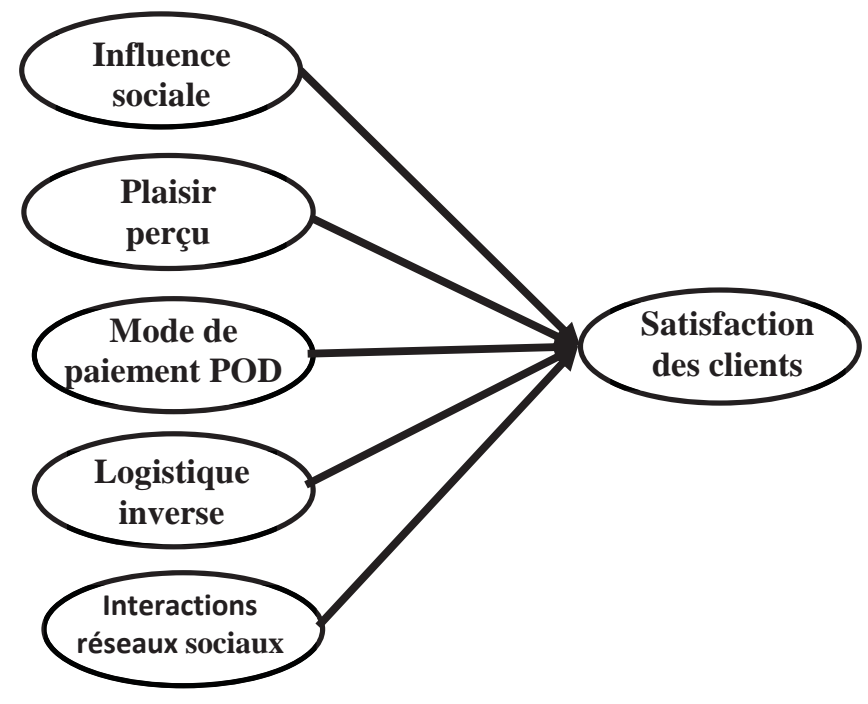

Figure 1 - Modèle proposé

\section{Méthodologie de recherche}

\subsection{Instruments de sondage}

La population visée par cette recherche est constituée des gens effectuant des achats en ligne en Inde. Cette étude a été menée dans les États de l'Inde du Nord, notamment Jammu-et-Cachemire, Himachal Pradesh, Penjab, Chandigarh, Haryana, Delhi et Uttar Pradesh. Les États de l'Inde du Nord représentent un assortiment de traditions et de coutumes (Singh et al., 2017). Ils comprennent beaucoup de jeunes et moins de femmes que d'hommes.

En raison de la percée du Web, les acheteurs de l'Inde du Nord ont connu un grand changement dans leur mode de vie et leurs habitudes d'achat au cours des dernières années; ils préfèrent l'achat en ligne du point de vue utilitaire et hédoniste (Tandon et al., 2017). Les Indiens du Nord ont un bon pouvoir d'achat, en comparaison avec le reste du pays, et ils l'utilisent en grande partie en effectuant des achats en ligne. Cette tendance croissante nous a motivés à examiner et à analyser la perception des acheteurs de l'Inde du Nord.
Le barème du mode de paiement par POD comprend cinq éléments provenant de l'étude précédente de Tandon et Kiran (2019). Les éléments des interactions sur les réseaux sociaux de Duffett (2015) ont été retenus, considérant que les éléments de la logistique inverse sont de nouveaux éléments du barème. Les éléments sur la satisfaction des clients des précédentes études de Tandon et al. (2017 ont été retenus. Les éléments du barème ont été adaptés à l'achat en ligne. Tous les éléments ont été mesurés à l'aide d'une échelle de Likert à cinq points allant de « tout à fait en accord » à «fortement en désaccord ».

\subsection{Procédure de collecte de données}

Pour améliorer la clarté du questionnaire, la version préliminaire a été distribuée à un groupe pilote d'universitaires et de doctorants. Le groupe pilote a répondu aux questions et a suggéré des modifications mineures dans la formulation des éléments. Ses suggestions ont été intégrées au questionnaire en ajoutant et en retirant quelques questions. Au total, 650 réponses ont été reçues par le biais du sondage. Toutefois, après un examen attentif, seuls 424 questionnaires ont été retenus pour une analyse plus approfondie. 
Une comparaison a été faite entre les répondants hâtifs et tardifs pour remédier au biais de non-réponse (Armstrong et Overton, 1977). Aucune différence significative n'a été constatée, ce qui indique l'absence d'un biais de non-réponse (voir tableau 1). Ainsi, l'échantillon final de 424 répondants peut être considéré comme étant représentatif de l'ensemble de la population.

\begin{tabular}{|c|c|c|c|c|c|c|c|}
\hline $\begin{array}{l}\text { Types de } \\
\text { répondants } \\
(\mathrm{N}=424)\end{array}$ & & $\begin{array}{c}\text { Satisfaction } \\
\text { des clients }\end{array}$ & $\begin{array}{l}\text { Mode de } \\
\text { paiement } \\
\text { par POD }\end{array}$ & $\begin{array}{c}\text { Interactions } \\
\text { sur les } \\
\text { réseaux } \\
\text { sociaux }\end{array}$ & $\begin{array}{l}\text { Plaisir } \\
\text { perçu }\end{array}$ & $\begin{array}{c}\text { Influence } \\
\text { sociale }\end{array}$ & $\begin{array}{r}\text { Logistiq1 } \\
\text { inverse }\end{array}$ \\
\hline \multirow{2}{*}{$\begin{array}{l}\text { Répondants } \\
\text { hâtifs } \\
(n=303)\end{array}$} & Moyenne & 4,04 & 3,82 & 3,64 & 3,87 & 4,11 & 3,93 \\
\hline & S. D. & 0,94 & 0,88 & 1,13 & 0,91 & 0,92 & 0,99 \\
\hline \multirow{2}{*}{$\begin{array}{l}\text { Répondants } \\
\text { tardifs } \\
(\mathrm{n}=121)\end{array}$} & Moyenne & 4,01 & 3,80 & 3,64 & 3,82 & 4,09 & 3,86 \\
\hline & S. D. & 0,93 & 0,86 & 1,15 & 0,80 & 0,88 & 0,98 \\
\hline
\end{tabular}

Tableau 1 - Biais de non-réponse

Le tableau 2 présente les caractéristiques démogra- un bon niveau d'instruction, c'est-à-dire diplômé phiques des clients sondés. Le profil des répon- $(35,1 \%)$ ou ayant terminé des études supérieures dants révèle qu'un nombre considérable possède (50,8 \%).

\begin{tabular}{lcc}
\hline Caractéristiques démographiques & $\mathbf{N}^{\text {bre }}$ & $\%$ \\
\hline Genre & & \\
Homme & 254 & 60 \\
Femme & 170 & 40 \\
Niveau d'études & & \\
Premier cycle & 60 & 14,1 \\
Diplômé & 149 & 35,1 \\
Études supérieures & 215 & 50,8 \\
Âge & & \\
18 à 30 ans & 221 & 52 \\
31 à 44 ans & 118 & 28 \\
Plus de 45 ans & 85 & 20 \\
Type de client & & \\
Étudiant & 56 & 13,2 \\
Travailleur autonome & 75 & 17,7 \\
Service & 293 & 69,1 \\
\hline Note. N 424 & &
\end{tabular}

Tableau 2 - Répartition de fréquence du profil démographique des répondants 
Le tableau 3 illustre quant à lui l'utilisation de

l'Internet et les habitudes d'achat en ligne des

424 répondants.

\begin{tabular}{|c|c|c|}
\hline Caractéristiques & $\mathbf{N}^{\text {bre }}$ & $\%$ \\
\hline \multicolumn{3}{|c|}{ Nbre d'heures par semaine consacrées à Internet } \\
\hline Moins de 10 heures & 45 & 10,6 \\
\hline De 11 à 20 heures & 139 & 32,8 \\
\hline Plus de 20 heures & 240 & 56,6 \\
\hline \multicolumn{3}{|c|}{$N^{\text {bre }}$ d'heures par mois consacrées à l'achat en ligne } \\
\hline Moins de 5 heures & 212 & 42,4 \\
\hline De 6 à 11 heures & 227 & 45,4 \\
\hline Plus de 11 heures & 61 & 12,2 \\
\hline \multicolumn{3}{|c|}{$N^{\text {bre }}$ de produits achetés durant les 3 derniers mois } \\
\hline Moins de 5 & 94 & 18,8 \\
\hline De 6 et 10 & 215 & 43,0 \\
\hline Plus de 10 & 191 & 38,2 \\
\hline \multicolumn{3}{|c|}{ Nbre d'années d'expérience d'achat en ligne } \\
\hline Moins de 3 ans & 54 & 12,7 \\
\hline De 4 à 6 ans & 69 & 16,3 \\
\hline Plus de 6 ans & 301 & 71 \\
\hline \multicolumn{3}{|c|}{ Mode de paiement préféré } \\
\hline POD & 332 & 66,4 \\
\hline Carte de crédit & 63 & 12,6 \\
\hline Carte de débit & 105 & 21 \\
\hline
\end{tabular}

Tableau 3 - Utilisation d'Internet et habitudes d'achat en ligne

\section{Analyse des données}

\subsection{Fiabilité et validité}

Pour évaluer la fiabilité et la validité du modèle de mesure proposé, une analyse factorielle confirmatoire (AFC) a été effectuée. Les résultats de l'AFC (voir tableau 4) indiquent que les charges normalisées de toutes les variables incluses sont significatives. L'instrument démontre la preuve de validité convergente (variance moyenne extraite $>0,50$ en toute circonstance), la fiabilité composite (valeur de $>0,70$ en toute circonstance) et la validité discriminante. 


\begin{tabular}{|c|c|c|c|c|c|c|}
\hline Variables & Éléments & $\begin{array}{l}\text { Estimation } \\
\text { normalisée }\end{array}$ & $\begin{array}{l}\text { Erreur } \\
\text { type }\end{array}$ & $\begin{array}{c}\text { Ratio } \\
\text { critique }\end{array}$ & $\begin{array}{l}\text { Écart moyen } \\
\text { extrait }\end{array}$ & $\begin{array}{c}\text { Fiabilité } \\
\text { composite }\end{array}$ \\
\hline \multirow{3}{*}{$\begin{array}{l}\text { Logistique inverse } \\
\text { (REL) }\end{array}$} & REL1* & 0,841 & & & \multirow[t]{3}{*}{0,647} & \multirow[t]{3}{*}{0,846} \\
\hline & REL2 & 0,830 & 0,056 & 17,376 & & \\
\hline & REL3 & 0,739 & 0,054 & 15,727 & & \\
\hline \multirow{3}{*}{$\begin{array}{l}\text { Réseaux sociaux } \\
(\mathrm{SOM})\end{array}$} & SOM3 & 0,701 & 0,047 & 15,467 & \multirow[t]{3}{*}{0,662} & \multirow[t]{3}{*}{0,853} \\
\hline & SOM4* & 0,841 & & & & \\
\hline & SOM5 & 0,887 & 0,05 & 19,629 & & \\
\hline \multirow[t]{3}{*}{ Plaisir perçu (PEJ) } & PEJ1* & 0,745 & & & \multirow[t]{3}{*}{0,581} & \multirow[t]{3}{*}{0,806} \\
\hline & PEJ2 & 0,714 & 0,125 & 12,818 & & \\
\hline & PEJ3 & 0,824 & 0,108 & 13,192 & & \\
\hline \multirow{3}{*}{$\begin{array}{l}\text { Influence sociale } \\
\text { (SOI) }\end{array}$} & SOI1* & 0,903 & & & \multirow[t]{3}{*}{0,819} & \multirow[t]{3}{*}{0,932} \\
\hline & SOI2 & 0,929 & 0,035 & 29,233 & & \\
\hline & $\mathrm{SOI3}$ & 0,883 & 0,039 & 26,739 & & \\
\hline \multirow{4}{*}{$\begin{array}{l}\text { Paiement à la } \\
\text { livraison (POD) }\end{array}$} & POD1* & 0,686 & & & \multirow[t]{4}{*}{0,537} & \multirow[t]{4}{*}{0,822} \\
\hline & POD3 & 0,784 & 0,107 & 13,127 & & \\
\hline & POD4 & 0,769 & 0,107 & 12,983 & & \\
\hline & POD5 & 0,686 & 0,08 & 13,837 & & \\
\hline \multirow{3}{*}{$\begin{array}{l}\text { Satisfaction des } \\
\text { clients (CUS) }\end{array}$} & CUS1* & 0,797 & & & \multirow[t]{3}{*}{0,644} & \multirow[t]{3}{*}{0,844} \\
\hline & CUS2 & 0,800 & 0,063 & 16,534 & & \\
\hline & CUS3 & 0,810 & 0,059 & 16,719 & & \\
\hline
\end{tabular}

Tableau 4 Validité du modèle de mesure

L'estimation de variance moyenne extraite (VME) de carrée de celle-ci que de tout autre concept (voir chaque concept est plus grande que la corrélation tableau 5) (Fornell et Larcker, 1981).

\begin{tabular}{l|cccccc}
\hline \multicolumn{1}{c}{ CUS } & POD & SOM & PEJ & SOI & REV \\
\hline CUS & $\mathbf{0 , 8 0 2}$ & & & & & \\
POD & $0,524^{* *}$ & $\mathbf{0 , 7 3 2}$ & & & & \\
SOM & $0,571^{* *}$ & $0,527^{* *}$ & $\mathbf{0 , 8 1 3}$ & & & \\
PEJ & $0,370^{* *}$ & $0,447^{* *}$ & $0,415^{* *}$ & $\mathbf{0 , 7 6 2}$ & & \\
SOI & $0,276^{* *}$ & $0,371^{* *}$ & $0,255^{* *}$ & $0,478^{* *}$ & $\mathbf{0 , 9 0 4}$ & \\
REV & $0,502^{* *}$ & $0,438^{* *}$ & $0,414^{* *}$ & $0,409^{* *}$ & $0,358^{* *}$ & $\mathbf{0 , 8 0 4}$ \\
\hline
\end{tabular}

Note. CUS : satisfaction des clients; POD : paiement à la livraison; SOM : interactions sur les réseaux sociaux; $\mathrm{PEJ}$ : plaisir perçu; $\mathrm{SOI}$ : influence sociale et $\mathrm{REV}$ : logistique inverse.

** La corrélation est significative au niveau 0,01 (bilatéral). Les données en gras représentent la racine carrée de la VME.

Tableau 5 - Matrice de corrélation des variables 


\subsection{Modèle structurel}

Ce modèle d'hypothèse a été estimé séparément sur l'ensemble des variables indépendantes et sur la variable dépendante de la satisfaction des clients (voir tableau 6).

\begin{tabular}{|c|c|c|c|c|c|c|c|}
\hline & & & $\begin{array}{c}\text { Charge } \\
\text { normalisée }\end{array}$ & $\begin{array}{l}\text { Erreur } \\
\text { type }\end{array}$ & $\begin{array}{c}\text { Ratio } \\
\text { critique }\end{array}$ & $\begin{array}{c}\text { Valeur } \\
\mathrm{p}\end{array}$ & Résultat \\
\hline Plaisir perçu(1) & $\rightarrow$ & $\begin{array}{l}\text { Satisfaction } \\
\text { des clients }\end{array}$ & 0,72 & & & & Soutenu \\
\hline $\begin{array}{l}\text { Paiement à la } \\
\text { livraison }\end{array}$ & $\rightarrow$ & $\begin{array}{l}\text { Satisfaction } \\
\text { des clients }\end{array}$ & 0,377 & 0,092 & 5,003 & $* * *$ & Soutenu \\
\hline $\begin{array}{l}\text { Interactions sur les } \\
\text { réseaux sociaux }\end{array}$ & $\rightarrow$ & $\begin{array}{l}\text { Satisfaction } \\
\text { des clients } \\
\text { Satisfaction }\end{array}$ & 0,137 & 0,092 & 1,139 & 0,072 & Non soutenu \\
\hline Influence sociale & $\rightarrow$ & $\begin{array}{l}\text { des clients } \\
\text { Satisfaction }\end{array}$ & 0,376 & 0,028 & 9,208 & $* * *$ & Soutenu \\
\hline Logistique inverse & $\rightarrow$ & des clients & 0,27 & 0,05 & 4,712 & $* * *$ & Soutenu \\
\hline
\end{tabular}

Tableau 6 - Modèle structurel des facteurs de motivation de l'achat en ligne

\section{Discussion et conclusion}

\subsection{Discussion}

Cette recherche visait à comprendre les facteurs influençant les commerçants en ligne à cibler le plus grand potentiel inexploité et les budgets inaccessibles dans les régions rurales et au-delà des zones urbaines de deuxième catégorie, lesquelles réfèrent à des agglomérations d'au moins un million d'habitants comme les capitales des différentes provinces indiennes et les centres industriels. Les commerçants en ligne peuvent définir et surmonter les défis en créant une infrastructure dans les régions éloignées qui facilite la livraison des produits par POD et qui garantit une protection adéquate sur les retours.

Le modèle de recherche a validé et a défini l'impact du plaisir perçu, du mode de paiement par POD, des interactions sur les réseaux sociaux, de l'influence sociale et de la logistique inverse sur la satisfaction des clients qui font des achats en ligne.

Le plaisir perçu est apparu comme étant le plus fort (charge factorielle normalisée $=0,72 ; \mathrm{p}<0,000$ ) et 'indice le plus significatif de la satisfaction des clients, ce qui est cohérent avec les précédentes études publiées (Tandon et Kiran, 2019; Venkatesh et al., 2012; Hwang, 2010;). Cela indique que le processus de recherche de nouveaux produits semble agréable pour les acheteurs et a ainsi une influence sur l'achat en ligne. Se sentir heureux et excité en parcourant les sites d'achat satisfera l'acheteur.

Puis, le mode de paiement par POD est également apparu comme un indice significatif (charge normalisée $=0,377 ; \mathrm{p}<0,000)$ de la satisfaction des clients. Cette conclusion concorde avec les études précédentes (Tandon et Kiran 2019; Chiejina et Olamide, 2014). Puisque ce mode de paiement est fortement privilégié par les acheteurs en ligne dans les pays en développement comme l'Inde, un effort est exigé de la part des cybercommerçants pour l'étendre au plus grand nombre de codes postaux. Les chercheurs peuvent également l'étudier plus en profondeur dans des territoires et des contextes culturels différents.

Ces indices sont suivis de l'influence sociale et de la logistique inverse. Le rapport d'influence sociale est apparu comme un indice significatif (charge normalisée $=0,376 ; \mathrm{p}<0,000)$, qui est cohérent avec les précédentes études de Yaprakli et al. (2013) et de Venkatesh et al. (2012), mais incohérent avec l'étude de Baptista et Oliveira (2015). La logistique inverse apparait également comme étant significative dans l'étude, mais obtient la charge factorielle la plus basse (charge normalisée $=0,27 ; \mathrm{p}<0,000$ ). Cette conclusion est cohérente avec les précédentes études publiées (Yu et Wang, 2008; Altug et Aydinliyim, 2016). Même si la logistique inverse apparaît comme étant la variable significative la plus faible, des efforts déployés sur la logistique inverse et sur des retours sans tracas pourraient aider à générer de la confiance 
et à surmonter l'appréhension chez les Indiens concernant l'achat en ligne. Par conséquent, il est absolument nécessaire de se concentrer sur le plaisir perçu, sur le POD, sur l'influence sociale et sur la logistique inverse pour encourager l'achat en ligne.

Étonnamment, les interactions sur les réseaux sociaux sont apparues comme étant négligeables. Cette conclusion est incohérente avec les précédentes études publiées (Muda et al., 2016; Ioanăs et Stoica, 2014;
Tandon et Kiran, 2019;). Une explication possible serait que les répondants du groupe sondé ne sont peut-être pas des utilisateurs actifs de plateformes de réseaux sociaux.

Mettre véritablement l'accent sur les concepts validés pourrait aider à générer de la confiance en soi et à invalider l'appréhension chez les Indiens en ce qui concerne l'achat en ligne.

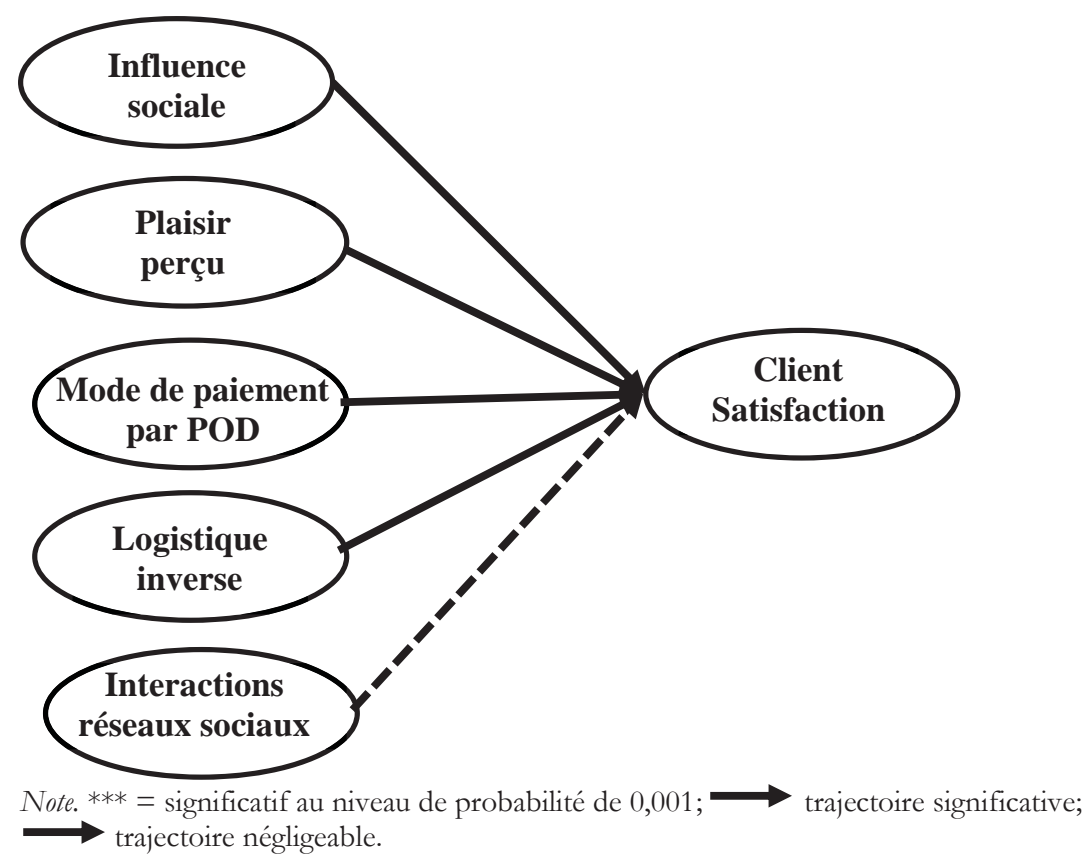

Figure 2-Relations des trajectoires présentant la relation entre les variables

\subsection{Contributions de l'étude}

La principale contribution de cette étude repose sur la validation du rôle des facteurs de motivation de l'achat en ligne, qui sont extrêmement importants pour l'adoption, la diffusion et la satisfaction envers toute technologie. L'achat en ligne est en voie de changer la donne pour le développement du cybercommerce dans les villes de deuxième catégorie et les zones rurales de moindre catégorie. Ce changement structurel se traduira par des développements infrastructurels puisque les commerçants en ligne exigeront des itinéraires et des manutentionnaires de produits efficaces pour la dernière étape de livraison non couverte chez les clients ruraux.

Une autre contribution théorique importante est la validation des indices (plaisir perçu, influence sociale, logistique inverse, mode de paiement par POD et interactions sur les réseaux sociaux) pour comprendre leur relation avec la satisfaction des clients. En raison de recherches limitées sur les mécanismes de paiement, la contribution du mode de paiement par POD est reconnue en tant que vecteur de confiance entre les clients et les cybercommerçants pour l'achat en ligne. De plus, la logistique inverse, qui peut être considérée comme étant une partie prédestinée de l'achat en ligne, a été validée pour la première fois en lien avec la satisfaction des clients. Une mauvaise expérience de retour de produit réduit les chances qu'un client effectue un rachat. Ainsi, les retours sans tracas jouent un rôle significatif pour la détermination des attentes des clients et améliorent l'expérience globale d'achat. L'importance de mettre en place un processus de logistique inverse devient donc évidente.

La conclusion de cette recherche a également des retombées importantes pour les commerçants en 
ligne. Mettre l'accent sur les indices qui ont émergé de l'étude n'est pas seulement crucial pour les cybercommerçants en Inde, mais aussi pour les autres pays en développement. Les cybercommerçants doivent d'abord améliorer la dimension agréable de l'expérience d'achat en ligne et poursuivre avec le mode de paiement par POD, avec la logistique inverse et avec un bouche-à-oreille positif par le biais des normes sociales.

\section{Limites et pistes de recherche}

Tout en contribuant à la théorie de commercialisation existante et à la littérature, notre étude possède quelques limites, que nous reconnaissons en tant qu'indications pour les recherches à venir. Une importante limite est le manque de généralisation de ses conclusions. Puisque les données ont été recueillies dans les États de l'Inde du Nord, les conclusions peuvent ainsi n'être pertinentes que pour les États de l'Inde du Nord. Cette recherche pourrait être élargie à l'Inde de l'Est, de l'Ouest et du Sud, car non seulement l'exposition à la technologie est diverse, mais les infrastructures logistiques le sont également. Pour tester la validité des conclusions, cette étude pourrait s'étendre aux autres pays en développement.

La seconde limite de cette étude est que les variables pertinentes telles que l'innovation personnelle, le risque perçu, la qualité du site web et les politiques gouvernementales devraient être comprises. Ainsi, des recherches futures pourraient également inclure d'autres facteurs de motivation intrinsèque et extrinsèque afin d'adopter un large éventail de technologies. L'étude laisse aussi place à la possibilité de valider l'impact modéré des variables catégorielles telles que l'âge et le genre.

\section{RÉFÉRENCES}

Altug, M. S. et Aydinliyim, T. (2016). Counteracting strategic purchase deferrals: The impact of online retailers' return policy decisions. Manufacturing and Service Operations Management, 18(3), 376-392. https://doi.org/10.1287/msom.2015.0570

Armstrong, J. S. et Overton, T. S. (1977). Estimating nonresponse bias in mail surveys. Journal of Marketing Research, 14(3), 396-402. https://doi.org/10.1177\%2F002224377701400320

Ashman, R., Solomon, M. R. et Wolny. J. (2015). An old model for a new age: Consumer decision making in participatory digital culture. Journal of Consumer Behavior, 14(2), 127-146. https://doi.org/10.1362/147539215X14373846805743

Baptista, G. et Oliveira, T. (2015). Understanding mobile banking: The unified theory of acceptance and use of technology combined with cultural moderators. Computers in Human Behavior, 50, 418-430. https://doi.org/10.1016/j.chb.2015.04.024

Brown, S. A. et Venkatesh, V. (2005). Model of adoption of technology in households: A baseline model test and extension incorporating household life cycle. MIS Quarterly, 29(3), 399-426.

Chang, C. C. et Chen, C. W. (2015). Examining hedonic and utilitarian bidding motivations in online auctions: Impacts of time pressure and competition. International Journal of Electronic Commerce, 19(2), 39-65. https://doi.org/10.1080/10864415.2015.979476

Chiejina, C. et Olamide, S. E. (2014). Investigating the significance of the « Pay on Delivery » option in emerging prosperity of the Nigerian e-commerce sector. Journal of Marketing and Management, 5(1), 120-135.

Crisafulli, B. et Singh, J. (2017). Service failures in e-retailing: Examining the effects of response time, compensation, and service criticality. Computers in Human Behavior, 77, 413-424. https://doi.org/10.1016/j.chb.2017.07.013

Dharmawirya, M., et Smith, B.A. (2012). Analysis of consumer repurchase intention towards online shopping in Indonesia's online retail business market. International Journal of e-Education, e-Business, e-Management and e-Learning, 2(3), 202-205.

Deci, E. L. et Ryan, R. M. (1985). Intrinsic motivation and self-determination in human behavior. New York, NY: Plenum.

Deci, E. L. et Ryan, R. M. (2000). The « what» and « why » of goal pursuits: Human needs and the self-determination of behavior. Psychological Inquiry, 11(4), 227-268. https://doi.org/10.1207/S15327965PLI1104_01

Deloitte et Assocham India. (2014). Future of e-commerce: Uncovering innovation. Mumbai, Inde: Deloitte Touche Tohmatsu India. Repéré à https://www2.deloitte.com/content/dam/Deloitte/in/Documents/technology-media-telecommunications/in-tmtfuture-of-e-commerce-noexp.pdf

Duffett, R. G. (2015). Facebook advertising's influence on intention-to-purchase and purchase amongst Millennials. Internet Research, 25(4), 498-526. https://doi.org/10.1108/IntR-01-2014-0020 
Fornell, C. et Larcker, D. F. (1981). Evaluating structural equation models with unobservable variables and measurement error. Journal of Marketing Research, 18(1), 39-40. https://doi.org/10.1177/002224378101800104

Gagné, M. et Deci, E. L. (2005). Self-determination theory and work motivation. Journal of Organizational Behavior, 26(4), 331-362. https://doi.org/10.1002/job.322

Gao, W., Liu, Y., Liu, Z. et Li, J. (2018). How does presence influence purchase intention in online shopping markets? An explanation based on self-determination theory. Behavior and Information Technology, 37(8), 786-799. https://doi.org/10.1080/0144929X.2018.1484514

Hajli, M. N. (2014). A study of the impact of social media on consumers. International Journal of Market Research, 56(3), 388-404. https://doi.org/10.1080/0144929X.2018.1484514

Harris, C. et Martin, K. B. (2014). The reverse logistics of online retailing, its evolution and future directions. Journal of System and Management Science, 4(2), 1-14. Repéré à http://www.aasmr.org/jsms/Vol4/No.2/JSMS_VOL4_NO2_001.pdf

Hew, T. S. et Kadir, S. L. S. A. (2017). Applying channel expansion and self-determination theory in predicting use behavior of cloud-based VLE. Behavior and Information Technology, 36(9), 875-896. https://doi.org/10.1080/0144929X.2017.1307450

Hwang, Y. (2010). The moderating effects of gender on e-commerce systems adoption factors: An empirical investigation. Computers in Human Behavior, 26, 1753-1760. https://doi.org/10.1016/j.chb.2010.07.002

IBEF Report (2018). Retail Industry in India. Repéré à : https://www.ibef.org/archives/detail/b3ZlcnZpZXcmMzc2MzYmMzc1

Ioanăs, E. et Stoica, I. (2014). Social media and its impact on consumers behavior. International Journal of Economic Practices and Theories, 4(2), 295-303. Repéré à https://www.researchgate.net/publication/313421625_Social_Media_and_its_Impact_on_ Consumers_Behavior

Kempe, D., Kleinberg, J. et Tardos, É. (2003, août). Maximizing the spread of influence through a social network. Dans Proceedings of the 9th ACM SIGKDD International conference on Knowledge discovery and data mining (p. 137-146). Washington, D.C. Repéré à https://www.cs.cornell.edu/home/kleinber/kdd03-inf.pdf

Kim, E. et Drumwright. M. (2016). Engaging consumers and building relationships in social media: How social relatedness influences intrinsic vs. extrinsic consumer motivation. Computers in Human Behavior, 63, 970-979. https://doi.org/10.1016/j.chb.2016.06.025

Kumar, A. et Kashyap, A. N. (2018). Leveraging utilitarian perspective of online shopping to motivate online shoppers. International Journal of Retail and Distribution Management, 46(3), 247-263. https://doi.org/10.1108/IJRDM-08-2017-0161

Li, Y., Xu, L. et Li, D. (2013). Examining relationships between the return policy, product quality, and pricing strategy in online direct selling. International Journal of Production Economics, 144(2), 451-460. https://doi.org/10.1016/j.ijpe.2013.03.013

Martin, K. D. et Hill, R. P. (2012). Life satisfaction, self-determination, and consumption adequacy at the bottom of the pyramid. Journal of Consumer Research, 38(6), 1155-1168. https://doi.org/10.1086/661528

Muda, M., Mohd, R. et Hassan, S. (2016). Online purchase behavior of generation Y in Malaysia. Procedia Economics and Finance, 37, 292-298. https://doi.org/10.1016/S2212-5671(16)30127-7

Nielsen. (2017). What's next in e-commerce understanding the omni-channel consumer. Repéré à https://www.nielsen.com/us/en/insights/ report/2017/whats-next-in-e-commerce-2/\#

Oghazi, P., Karlsson, S., Hellström, D. et Hjort, K. (2018). Online purchase return policy leniency and purchase decision: Mediating role of consumer trust. Journal of Retailing and Consumer Services, 41, 190-200. https://doi.org/10.1016/j.jretconser.2017.12.007

Oliver, R. (1993). Cognitive, affective, and attribute bases of the satisfaction response. Journal of Consumer Research, 20(3), 418-430. https://doi.org/10.1086/209358

Pei, Z., Paswan, A. et Yan, R. (2014). E-tailer's return policy, consumer's perception of return policy fairness and purchase intention. Journal of Retailing and Consumer Services, 21(3), 249-257. https://doi.org/10.1016/i.jretconser.2014.01.004lu

Sheikh, Z., Yezheng, L., Islam, T., Hameed, Z. et Khan, I. (2019). Impact of social commerce constructs and social support on social commerce intentions. Information Technology and People, 32(1), 68-93. https://doi.org/10.1108/ITP-04-2018-0195

Singh, N., Srivastava, S. et Sinha, N. (2017). Consumer preference and satisfaction of M-wallets: A study on North Indian consumers. International Journal of Bank. Marketing, 35(6), 944-965. https://doi.org/10.1108/IJBM-06-2016-0086 
Slyke, V. C., Belanger, F. et Hightower, R. (2005, mars). Understanding gender-based differences in consumer e-commerce adoption. Dans Proceedings of the 2005 Southern Association of Information Systems Conference (p. 24-29). (s. 1.).

Repéré à https://aisel.aisnet.org/sais2005/5

Sushma, U. N. (2017, 29 septembre). Morgan Stanley explains why India's e-commerce market is a hot investment opportunity. Quarts India. Repéré à https:/ / qz.com/india/1089559/morgan-stanley-explains-why-indias-e-commerce-market-is-a-hot-investmentopportunity

Tandon, U. et Kiran, R. (2019). Factors impacting customer satisfaction: An empirical investigation into online shopping in India. Journal of Information Technology Case and Application Research, 21(1), 13-34. https://doi.org/10.1080/15228053.2019.1609779

Tandon, U., Kiran, R. et Sah, A. (2017). Customer satisfaction as mediator between website service quality and repurchase intention: An emerging economy case. Service Science, 9(2), 106-120. https://doi.org/10.1287/serv.2016.0159

Tavana, M., Shabani, A. et Singh, R. (2019). The impact of interwoven integration practices on supply chain value addition and firm performance. Journal of Industrial Engineering International, 15(1), 39-51.

Thong, J. Y. L., Hong, S. J. et Tam, K. Y. (2006). The effects of post-adoption beliefs on the expectation-confirmation model for information technology continuance. International Journal of Human-Computer Studies, 64(9), 799-810. https://doi.org/10.1016/j.ijhcs.2006.05.001

Venkatesh, V., Morris, M. G., Davis, G. B. et Davis, F. D. (2003). User acceptance of information technology: Towards a unified view. MIS Quarterly, 27(3), 425-478. https://doi.org/10.2307/30036540

Venkatesh, V. et Speier, C. (2000). Creating an effective training environment for enhancing telework. International Journal of Human-Computer Studies, 52(6), 991-1005. https://doi.org/10.1006/ijhc.1999.0367

Venkatesh, V., Thong, J. Y. L. et Xu, X. (2012). Consumer acceptance and use of information technology: Extending the unified theory of acceptance and use of technology. MIS Quarterly, 36(1), 157-178. https://doi.org/10.17705/1jais.00428

Vijay, N. (2020, 3 janvier). View: The biggest challenge facing e-commerce sector in India \& how to overcome. The Economic Times. Repéré à https://economictimes.indiatimes.com/industry/services/retail/view-the-biggest-challenge-facing-ecommerce-sectorin-india-how-to-overcomeit/articleshow $/ 73076390 . \mathrm{cms} ?$ utm_source $=\% 20$ contentofinterest $\% \% 20 \% 20$ utm_ $\% 20$ medium=text\&utm_campaign=

White, C. (2015). The impact of motivation on customer satisfaction formation: A self-determination perspective. European Journal of Marketing, 49(11/12), 1923-1940. https://doi.org/10.1108/EJM-08-2014-0501

Yaprakli, S., Kalbakhani, E. et Rasouli, R. (2013). An application of UTAUT model for online shopping acceptance in Iran. Reef Resources Assessment and Management Technical Paper, 38(5), 237-244.

Yu, C. C. et Wang, C. S. (2008). A hybrid mining approach for optimizing returns policies in e-retailing. Expert Systems with Applications, 35(4), 1575-1582. https://doi.org/10.1016/j.eswa.2007.08.099

Zhang, K. Z., Zhao, S. J., Cheung, C. M. et Lee, M. K. (2014). Examining the influence of online reviews on consumers' decision-making: A heuristic-systematic model. Decision Support Systems, 67, 78-89. https://doi.org/10.1016/j.dss.2014.08.005 


\section{Annexe 1 - Éléments de l'échelle et leur source}

\section{Logistique inverse}

Je peux retourner le produit défectueux sans soucis.

La politique de retour permissive augmente ma confiance envers des achats en ligne répétés.

Le commerçant en ligne fournit de l'information détaillée sur le processus de logistique inverse.

Plaisir perçu (Venkatesh et al., 2012)

Faire des achats en ligne est une expérience excitante pour moi.

Faire des achats en ligne est amusant pour moi.

Je trouve que faire des achats en ligne est agréable.

\section{Mode de paiement par POD (Tandon et Kiran, 2019)}

Je crois que le POD est un mode de paiement fiable,

Je prévois payer grâce au mode de paiement par POD.

Je préfère acheter grâce au mode de paiement par POD.

Le mode de paiement par POD facilite et rend plus simple le retour de produits défectueux.

Le POD me donne confiance envers un rachat futur de produits.

Interactions sur les réseaux sociaux (Duffett, 2015)

La publication affichée sur la page Facebook décrit les fonctionnalités du produit annoncé.

Je visiterai la boutique en ligne de la marque pour acheter le produit présenté sur la page Facebook.

La publicité sur Facebook a une influence positive sur ma décision d'achat.

Influence sociale (Venkatesh et al., 2012)

Les gens qui sont importants pour moi croient que je devrais adopter l'achat en ligne.

Les gens qui ont une influence sur mon comportement croient que je devrais adopter l'achat en ligne.

Les gens dont l'opinion est importante pour moi croient que je devrais adopter l'achat en ligne.

Satisfaction des clients (Tandon et al., 2017)

Je suis satisfait de mon achat.

Je suis satisfait du mode de paiement par POD.

Je suis satisfait de la qualité du produit reçu grâce à un achat en ligne. 